\title{
A Theoretical Approach to the Effective Decision-Making Process
}

\author{
Federico de Andreis \\ Università Giustino Fortunato, Benevento, Italy \\ Email: f.deandreis@unifortunato.eu
}

How to cite this paper: de Andreis, F. (2020) A Theoretical Approach to the Effective Decision-Making Process. Open Journal of Applied Sciences, 10, 287-304. https://doi.org/10.4236/ojapps.2020.106022

Received: May 18, 2020

Accepted: June 13, 2020

Published: June 16, 2020

Copyright $\odot 2020$ by author(s) and Scientific Research Publishing Inc. This work is licensed under the Creative Commons Attribution International License (CC BY 4.0).

http://creativecommons.org/licenses/by/4.0/

(c) (i) Open Access

\begin{abstract}
This research develops and elaborates studies done for a contribution to the 2019 PIC International Conference 2019 in Malta, about the decision-making process. Decision-making is the act of choosing between two or more courses of action. In the wider process of problem-solving, decision-making involves choosing between possible solutions to a problem, and these decisions can be made through either an intuitive or reasoned process, or a combination of the two. The study of decision-making processes, to be understood as the role of human factors, becomes particularly interesting in complex organizations. This research aims to analyze how an effective team, within organizations, can develop a more correct and effective decision-making, in order to get an optimal solution, overcoming the typical uncertainty. The paper describes the point of departure of decision in complex, time-pressured, uncertain, ambiguous and changing environments. The use of a leading case (the Tenerife air accident, 1977), will lead us to the desired results, i.e. to demonstrate how an effective decisional process, including team dynamics, can be useful to reduce the risk, present in all decisions, and reduce errors. The case of Tenerife air disaster, confirm our research. In that case, in fact, the group dynamics prove not to have worked. Thus, we can state that if a team approach had been followed instead of a more individual one, the results would probably have been different. The central belief of the research, is that classic decision theory could benefit from a team approach, which reduces the risk that a decision may lead to undesirable consequences. As demonstrated with the case study, within organizations, the decision-making is not a solitary action. Decisions, in fact, are made within a team and in order to be able to function effectively in a group, and manage group situations, there are essential skills. The team can then become a resource for the decisional process and problem solving, but it is necessary to understand the dynamics.
\end{abstract}

\section{Keywords}

Decision-Making, Human Behavior, Problem Solving, Management, 


\section{Introduction}

The process of taking a decision also takes place without it being explicitly highlighted that there is an opportunity to take a decision.

This leads us to a definition of the decision-making process, i.e. that for a decision to be taken there must be at least two alternatives available.

The decision-making process, therefore, consists of choosing between alternative actions, to which a result corresponds.

We can say, however, that the decision-making process cannot begin until the existence of a given problem is explicitly recognized and resolved.

From this moment on, there is no a fixed path to follow to choose the best alternative, since problems can very rarely be solved by a sequential and linear approach but by a complex process.

In order to make the right decision, the team steps in to create a favorable environment. Within the organization, the teamwork helps to cultivate talents and qualifications and, while limiting the decisional power of the individual, through the group all members are free to take initiatives on various tasks (even the most difficult ones), to take risks and face the consequences [1].

The team, thus, helps to cultivate confidence in the skills and competencies of its members, to establish essential common goals that are in line with the organizations' purpose, through guidelines for all discussions and decision-making processes.

This research focuses on how team decisions facilitate the decisional process, reducing uncertainty and sharing risk; specifically, it will cover an investigation of the problem solving, i.e. the problem to be solved that triggers the need to make a decision, then it will focus on uncertainty, linked to decisions.

In the end, the analysis will lead us to understand how the team can reduce this uncertainty of the decisional process and with which tools it can intervene in the same to facilitate members to take a decision that is effective and affective for organizations.

\section{The Dimension of Problem Solving in the Decision-Making Process}

A problem can be defined as a situation in which an individual, "the solver", wishes to move from a given state (problem) to a desired one (solution), but cannot do so through instinctive action or learned behavior.

The term problem solving represents therefore the cognitive process used to analyze this situation/problem and find a solution.

We can therefore define problem solving as the most complex of all cognitive functions, since it consists in the ability to find a solution to any kind of prob- 
lems.

An effective problem solver must consequently be able to deal with any kind of situation and solve the difficulties it might encounter in the path that leads to the achievement of its objective.

This can therefore be done through multiple approaches, depending on the nature of the problem and the type of individuals or groups involved in it.

Among these, the most commonly used approach consists in implementing seven operations:

- description of the problem;

- analysis of the causes;

- identification of alternative solutions;

- verification of the validity of the various alternatives;

- choice of a solution;

- development of an implementation plan;

- monitoring of the plan until the desired result is obtained.

In other words, this process guides us in the first place to be able to firstly identify and define the problem; then to collect data and information; then again to formulate hypotheses of possible causes with the aim of verifying the validity of the various alternatives, searching for the most effective solution and corrective actions, finally after the implementation of an action plan it becomes necessary to verifying the results with the collection of monitoring data.

A correct process of analysis of a problem to which a solution must be offered, however, has at its basis some indications to follow. When collecting information, you should never accept anything as true, unless you obviously know it perfectly. It is then necessary to separate each difficulty or problem that is examined into as many small parts or sub-problems as possible and necessary, in order to better deal with them and then solve them. A further indication to follow is to lead the thoughts in order, starting from the simplest and easiest to know elements to go up, little by little and step by step, to the knowledge of the most complex ones.

Finally, problems must be considered with a general and comprehensive approach so as to ensure that nothing is omitted.

In the correct approach to problem solving, it is equally useful to be aware that there are no simple ways to solve complex problems. Each problem faced requires, decisions to be taken according to logical procedures.

- deduction "but-then"

A logical procedure by which a general truth (rule) can be derived from a particular one implicit in it.

All the beans in that bag are white-rule

These beans are from that bag-case

These beans are white-result (necessarily)

- induction "therefore"

A logical procedure in which the establishment of particular facts leads to 
statements or general rules.

These beans come from that bag-case

These beans are white-result

All the beans in that bag are white-rule (until proven otherwise)

- abduction "maybe"

A logical process that uses only a limited set of elements for which the decision maker creates the links and relationships between them, which could also be wrong.

These beans are white-result

All the beans in that bag are white-rule

These beans come from that bag-chance (maybe)

Contrary to a classic learning process that involves the use of schematic and automatic procedures, acquired previously and simply to be reapplied on similar problems, we can state that problem solving is based instead on cognitive operations able to offer an unexpected solution never reached before; this approach implies a structured reasoning aimed at solving a complex situation, which cannot be obtained with the automatic application of procedures already known nor with an instinctive or intuitive approach.

Problem solving activities are closely related to decision making, i.e. the process that leads to a decision taken by an individual or a group.

The decision, (taken both by an individual and by a group), and likewise the choice not to decide, implies voluntary and intentional behavior that follows a reasoning.

Usually the decision is made in order to solve a problem.

There is, however, a certain difference between deciding and solving a problem. In problem solving, the decision making act is always linked to the objective we want to achieve, while in decision making, the decision making act is represented by a reasoning of choice of the most suitable alternative-within a series of options.

The decision-making process can be considered as the result of mental processes (cognitive and emotional), which determine the selection of a course of action among different alternatives.

Considering that each decision making process produces a final choice and that making decisions usually requires the evaluation of at least two optionswhich differ with respect to different characteristics and elements-the selection of an option requires the person to make an overall assessment of the different alternatives, using:

- specific ways of searching and processing information;

- decision-making strategies.

However, in most cases, taking decisions means reasoning under conditions of uncertainty: it is not possible, in fact, predict with certainty the future outcome of the possible alternatives available, but at best it is only possible to estimate the probability of such outcomes [2].

Many studies on decision theory, in different areas, have defined the amounts 
of uncertainty and risk more specifically.

We can therefore define uncertainty as the lack of certainty, i.e. a limited state of knowledge, in which it is impossible to describe exactly the existing state, future results or more than one possible result.

The uncertainty can, however, still be measured and this is identified with a number of possible states in which results or probabilities are assigned to each possible state or result; his also includes the application of a continuous variable probability density function.

In the dimension of uncertainty, the risk also plays a fundamental role, i.e. the state of uncertainty in which some possible results have an undesirable effect or a significant loss and even in this case we can measure the age of possible losses.

Thus, we can state that uncertainty represents a fundamental dimension of today's society, to be considered in a radically distinct sense of risk but from which it cannot be properly separated, since it actually represents a lack of certainty, i.e. a limited state of knowledge in which it is impossible to describe exactly the existing state, future results or more of a possible result and thus the consequence of a lack of knowledge of facts that can be obtained.

In reality, therefore, the risk assumes the connotation of a state of uncertainty, in which some possible results have an undesirable effect or a significant loss.

For example, if you do not know whether it will rain tomorrow, you have an uncertain situation.

If you apply the probability of possible results using the weather forecast or even just a calibrated assessment of the probability, you could quantify the uncertainty.

Assuming you quantify the uncertainty with $90 \%$ chance of having a sunny day, if you are planning for example an outdoor event for the next day, you would have a $10 \%$ chance of rain, i.e. an undesirable condition. Moreover, if it were an outdoor business event and a sum equal to 100 thousand dollars were to be lost in the rain, this uncertainty could then be quantified with a $10 \%$ risk of losing 100 thousand dollars.

So we can then define this risk as EOL-Expected opportunity loss-loss of expected opportunities and calculate it through the following equation: possibility of loss by amount of losses (in the example $10 \% \times 100.000 \$=10.000 \$$ ).

\section{Uncertainty and Decision-Making}

The impact of uncertainty on the decisional process has its development in various areas.

Studies generally agree on the importance of two fundamental human motivations, such as the desire to reduce uncertainty and the desire to gain advantage (Bentham, 1948); these motivations are fundamental in decision making.

Contrary to early theories, which saw decision making linked to rational choice, today it is well known that human decisions are based as much on hedonic (pleasure) and emotional motivations as on rational motivations (Cabanac, 1992). 
Decision making processes are studied by different disciplines, from statistics to psychology to economics because the application of these studies is of enormous importance. In fact, the decision-making processes are transversal to different and multiple contexts, including, for example, decisions in the medical, political, economic, legal and judicial, organizational and business fields, in different types of emergency contexts, and so on.

Decision making is therefore closely related to probabilistic reasoning, i.e. an inferential inductive reasoning that allows us to estimate the probability that a given event within certain conditions can be realized.

With regard to theoretical models in the field of decision making there are two different approaches:

- a normative approach that focuses on rational choice theory. According to this theory, in conditions of uncertainty and risk, the options of choice are represented for individuals in terms of expected utility.

In other words, each possible alternative corresponds to a possible state of the attainable mode, which is associated with a corresponding probability value that it can actually be realized.

According to Von Neumann and Morgenstern (1944), who make a choice rationally, evaluate the utility corresponding to their choice and the probability that it will be realized (Pravettoni, Leotta, Russo, 2015).

Following the normative and rational choice theories, the human being reasons in terms of probabilistic and expected utility and applies the rules of statistics in a precise way.

- a descriptive approach. This opens the way for the descriptive approach of decision making models.

Along the same lines, the studies of Kahneman and Tversky (1974; 1981) found that people very often do not reason in statistical and rational terms, but would use the so-called heuristic strategies or cognitive bias, i.e. constructs based, outside of critical judgment, on erroneous or deformed perceptions, prejudices and ideologies; often used to make decisions quickly and effortlessly.

Very often, they are cognitive errors that have an impact on everyday life, not only on decisions and behavior, but also on thought processes.

The heuristics (from the Greek heurískein: to find, to discover) are, unlike bias, intuitive and quick mental procedures, mental shortcuts, which allow to build a generic idea on a subject without making too many cognitive efforts. They are fast strategies frequently used to reach conclusions quickly.

In particular Kahneman and Tversky have studied decision making processes under conditions of uncertainty and risk; their experiments have shown that during decision making processes-under conditions of uncertainty and riskthere are often systematic errors and cognitive bias that violate the assumptions of rational choice theory.

Such systematic errors, acting in a precise direction and in particular circumstances, are predictable, and by deepening the functioning of cognitive processes in decision making it is possible to identify their causes. 
Different categories of decision making strategies have been identified in the literature.

A first category of "compensatory" strategies includes for example the pros and cons model, according to which the individual evaluates the positive and negative attributes of the two alternatives, and the differences model, according to which the individual evaluates the difference between one option and the other.

The second category of decision making strategies consists of the "non-compensatory" models so that the different attributes are analyzed according to a restrictive and eliminatory criterion: the first negative aspect found, involves the elimination of the whole alternative.

In this procedure, therefore, the less pleasant alternatives are gradually eliminated.

In the decision making phase we can say that two strategies are normally and mainly adopted: that strategy to concentrating to the maximum time of decision in order to reduce the state of uncertainty and the one that refers to infinity the possibility of deciding.

Those who tend to prefer the first strategy, acquire less information, evaluate less hypothesis of solution and are satisfied with an acceptable result. It will therefore be geared towards a heuristic decision.

On the contrary, those who prefer to wait, without realizing that decisions often have a precise time limit, almost hope that at some point there is only one solution left.

The impression is that these people privilege algorithmic decisions, (going by exclusion until there is only one option left) but in reality, even if they make careful analyses and thoroughly evaluate the advantages and disadvantages of each solving hypothesis, they never get to complete the process.

In order to overcome these limits that the two different strategies pose to the decision making process, those who are leaning towards the heuristic mode, in order to resist from deciding in an almost instantaneous way, must begin to analyze more deeply even the small problems of daily life by listing a greater number of solutions.

Those who are leaning for the algorithmic mode, must give themselves a time limit, within which to guarantee the choice to be made, remembering that excessively delaying the decisions could make them lose even the remaining opportunities.

People who decide following the algorithmic mode can get lost, in fact, in one of the phases of decision making: problem setting (assessment of the situation), problem solving (identification of possible solutions), option choosing (choice of the best option), follow-up (evaluation of costs/benefits and results).

By analyzing specifically the decision-making process, we can therefore distinguish the four phases indicated above.

Describing in depth the first phase, we can define the problem setting as the 
step in which the collection of information takes place, aimed at the definition of the problem and at the analysis of the causal factors that generated the situation.

In other words, everything can be defined as situational awareness, that is the awareness of the situation that corresponds to the perception and understanding of all the factors that determine the surrounding situation.

In the mental sphere this process physically occurs at the level of short-term memory, also called working memory, which corresponds to the point of collection of information coming from sensory memories. These data, through very rapid mental associations, are manipulated and generate situational awareness.

It is now recognized and experienced how this brain area of work, under particular stress conditions, is reduced to process even significantly less data.

This explains why under certain stress conditions (reduce time available, too much workload, excessive workload, emergency situation) it is not always possible to collect all the necessary information. Many would, in fact, be easily available, but are not perceived or processed.

We must therefore highlight that the situational awareness involves being aware of what is happening nearby to understand how information, events and actions will impact on goals and targets, both immediately and in the near future.

An individual with an appropriate sense of situational awareness generally has a high degree of knowledge of the inputs and outputs of a system, an innate feeling for situations, people and events that occur because of the variables that the subject can control.

In studies on complex organizations and on effectiveness of work groups, the lack or inadequacy of situational awareness is therefore identified as one of the main factors in human error during the decision-making process.

Therefore, situational awareness is particularly important in work environments where the flow of information can be quite high and bad decisions can lead to negative (more or less serious) consequences. In this phase it is therefore extremely vital to move from an individual situational awareness to a team situational awareness, which can only be achieved by sharing the information held by individual members, since if something has escaped someone it is more difficult for everyone to escape it.

The second step of the decision making process is problem solving, a step in which possible options are defined.

This activity takes place in working memory, a model introduced by Baddeley and Hitch (1974) to describe more accurately the dynamics of short-term memory, which is a system for temporary storage and the first management/manipulation of information, i.e. a functional link between sensory perception and controlled action.

Also on working memory, similarly to what seen for situational awareness, the effects induced by stress (determined by the decision-making process) are simi$\operatorname{lar}[3]$. 
In these conditions it can be observed that the person is able to elaborate a smaller number of alternatives. therefore also for this phase it is important that the team is involved in the research and expansion of possible solutions.

The next step is that of option choosing, determined instead by a more careful evaluation of the advantages and disadvantages of each alternative and the choice of the one considered most convenient.

One of the elements that comes into play in this phase is the perception of risk. The assessment of risk, in fact, will be conditioned both by experience and by the acceptability of the level of risk itself. Under normal conditions a particular solution could be discarded as more dangerous.

However, under the stress of the decision-making process, higher levels of risk tend to be accepted and this means that the previously discarded hypothesis may now become one of the possible solutions.

The attitude of invulnerability then tends to prevail. The solution is in the awareness of these mechanisms, the ability to confront each other and the ability to observe in others those weak signals, possible symptoms of discomfort in the face of situations for which no danger is perceived.

In order to complete the phases of the decisional process, the effectiveness and feasibility of the decision taken must be evaluated, analyzed and, if it is necessary, improved.

This last step, the follow-up is not always present in decision making models, but extremely important.

During this period it is necessary to check that things go as planned and at the same time to be prepared for the "what if?", i.e. the presence of an alternative solution, which becomes vital when faced with complex, ambiguous situations or where the elements are constantly and rapidly changing.

One of the possible traps in these conditions is precisely the tendency to confirm (confirmation bias), so everything that happens goes on confirms reality rather than denies it.

As a demonstration of this, it has been noted that a substantial increase in the workload, and therefore in the operations to be done in the given time unit, can lead to a greater number of confirmation bias or cognitive errors.

\section{How Risk Perception Could Influence the Decision-Making Process}

Organizational decision making often occurs in the face of uncertainty about whether a decision maker's choices will lead to benefit or disaster. Risk is the potential that a decision will lead to a loss or an undesirable outcome. In fact, almost any human decision carries some risk, but some decisions are much more risky than others.

Risk and decision making are two inter-related factors in organizational management, and they are both related to various uncertainties.

Risk in fact, is the potential for a chosen action, decision taken or activity (in- 
cluding the choice not to act) to lead to a loss or undesirable event. The choice influences the result, thus the risks can be defined as the same potential losses that arise from any human behavior [4].

Some behaviors have a higher risk percentage.

Risk is present in modern societies and it is therefore related to human activities, as a result of the progress of modernization (Giddens). We can, in fact, distinguish the modern manufactured risks, which unlike in the past cannot only be associated with natural phenomena i.e. external risk.

The risk is therefore linked to the complexity of organizations and is distinguished from the danger (understood as external risk) that can be identified in the forces of nature (environmental, natural disasters, etc.) [5].

Progress and modernization have increased the forms of well-being but also the sources of risk. Risk differs from danger because it is precisely technological development that leads to an increase in risk, as it transforms the same dangers into risks, simply by creating the possibility of making decisions that previously could not be hypothesized.

When we have to make a decision, it is necessary then that the risk is identified, managed and controlled (risk analysis).

This potential condition/event that can modify an expected outcome can then be measured as the product between the probability that a specific event may occur and the extent of the damage (if any) associated with it.

In the organizations we introduce the value of the risk management which also considers in the above calculation the ability to predict the risk event and contain its consequences-through training, information, organization.

Assigning a value to the different risks can be difficult since despite a scientific calculus, each individual can assign a different value to a risk. This action clearly influences the decisional process, precisely because we are faced with a double dimension of risk: objective risk and subjective risk.

In other words we can say that the perception of risk is subject to objectivity, because we can calculate an objective possibility of occurrence, so we can state that the occurrence of an event is objectively determinable and to subjective, because, each individual has a different value of risk (risk perception) due to different parameters attributed to the risk itself (degree of belief) [6].

Interpreting Table 1, in summary, the unintentional exposure to a risk, the inability to control it, the unfamiliarity with the situation, the environment or similar recent experience will increase the perception of a particular risk; on the contrary, familiarity with the environment, voluntary exposure (i.e. the propensity to risk) or the idea that the risk can be controlled will decrease the perception of a risk.

For example, by analyzing the case of an airline pilot, we can say that it voluntarily exposes itself to the aeronautical risk, he is familiar with the environment and that he knows that he can control the plane; thus, his aeronautical risk perception will be negligible. 
Table 1. Risk perception.

\begin{tabular}{|c|c|c|}
\hline MAJOR RISK PERCEPTION & MINOR RISK PERCEPTION & EXAMPLE \\
\hline Involuntary exposure & voluntary exposure & $\begin{array}{l}\text { those who do not use a mobile } \\
\text { phone perceive exposure to radio } \\
\text { frequencies as a high risk. }\end{array}$ \\
\hline Inability to control & ability to control & $\begin{array}{l}\text { plane crash, for a pilot or a } \\
\text { passenger }\end{array}$ \\
\hline Unfamiliarity & familiarity & $\begin{array}{l}\text { familiarity with environment (e.g. } \\
\text { pilot) }\end{array}$ \\
\hline Recent experience & lack of experience & similar previous experience \\
\hline Dramatic risk & non dramatic risk & terrorist attack or car accident \\
\hline Disparity & equity & $\begin{array}{l}\text { when you are exposed to a risk } \\
\text { (electric fields) but have no benefits }\end{array}$ \\
\hline
\end{tabular}

On the contrary, a passenger-perhaps as a result of a negative experience, such as severe turbulence during a flight-will be able, at most, to perceive the risk as tolerable compared to the benefit of reducing the time to reach a place.

From an objective point of view, the calculation of the risk can be carried out by estimating the risk, to be understood as the definition of the probable seriousness of the damage and the probability of its occurrence.

When decisions have to be taken, it is therefore necessary to make an estimate of the possible risk resulting from the decision and consequently of the possibility of accepting a risk.

One of the possible mistakes one might fall into, is confirmation bias, i.e. convincing oneself that everything that happens is going to confirm reality rather than disprove it.

When a decision is being taken, especially at a time of stress or when time available is too short, individuals tend to want to confirm their acquired beliefs, with a tendency to be biased in examining ideas, focusing on a possibility and ignoring alternatives [7].

Rather than looking for all the relevant evidence to make a decision, individuals tend to construct questions so that they receive answers that support their ideas, seeking results that they'd expect if their assumptions were true, rather than those they'd get if they were false.

\section{Team Resources and Decision-Making}

Making a decision is not always easy, especially when you have to choose between two options that have both negative and positive implications.

The ability to assess the costs and benefits of an option is essential when individuals are faced with a decision that turns out to be satisfactory and rational.

Then situational awareness comes into play, which helps to have a clear and correct perception of the situation in which the decision is being taken, through the planning and assimilation of information from the many sources available; a very clear definition of the mental picture of what exactly has happened so far in 
similar situations and, finally, projecting forward the action taken, without acting instinctively.

In order to strengthen situational awareness, or recover it in case it is lost, in complex organizations teamwork takes place to support. Within the team, it is in fact easier to ask for support; to move away from the problem, increasing separation, to stabilize condition or parameters; to restore right priorities; to gain time, using it in the best possible way and finally to acquire additional information.

This starts precisely because in the decision-making process, thanks to teamwork, the contribution of all is of equal importance and that this allows to avoid those disagreements that would make cooperation and communication more difficult in case of need.

This can reach when the team explores the processes of creation, operation and maintenance of a working group and provides strategies and techniques to transform a working group itself, of any organizational context, in a functional team.

We can, in fact, define a team as a group of individuals who collaborate performing an activity. This is a group of two or more individuals who interact and depend on each other to achieve a common goal and it exists when these individuals define themselves as members and when its existence is recognized by at least one other individual. The simple, physical proximity does not constitute a team which must usually have defined tasks and activities, determined roles and high commitment of its members, because the team could work effectively when objectives and methods are clear and shared and when the whole group manages time define and respect roles, procedures and rules.

Working effectively in a team is very important for:

- outputs (results)

- quality

- moral (sense of responsibility)

- retention (conservation of the team itself)

Each team member has to make many decisions, decisions may be made in ad hoc ways or using processes that increase the likelihood of an effective choice. The processes through which decisions are reached may dramatically affect the quality of the decisions and team performance.

Thus, in order to make informed choices in selecting team decision making processes, teams need to learn how others have thought about decision-making processes.

How team members interact with each other creates an environment for decision-making. In examining environments, the focus is neither on the steps that a team might use to reach a decision nor how the various individual positions will be combined to reach a decision. The focus is on how team members listen to each other, how they formulate and ask questions of each other, and how they present their positions., i.e. an environment in which everyone on the team feels comfortable in sharing idea and proposing solutions raises the quality of the de- 
cisions.

Within a team, interdependence-i.e. a relationship of intimate connection and mutual dependence between several individuals, things, facts, phenomenabetween members constitutes the perception of individuals to be connected with others and an opportunity to get a positive decision environment.

The individual cannot succeed without being part of the team and likewise the team cannot succeed without the individual; this positive link of interdependence translates into the principle that the bond and the relationship with other people are a fundamental condition for the achievement of a result, an objective, or a reward [8].

In addition to creating an environment for effective decision-making and reaching consensus on methods for making decisions as a team, there are tools that can assist teams in formulating and reaching decisions, such as:

Brainstorming, with the goal of generating many options related to a specific purpose;

Affinity grouping, finalized to categorize the ideas generated by the brainstorming process, on the assumption that similar ideas can be grouped together to obtain a more organized picture of the ideas;

Multivoting, this is quick and easy and it is working as a process useful to allow a team to determine the higher priority and lower priority options from a set of alternatives.

Criteria matrix, that is useful to prioritize tasks, issues, alternatives, etc., to aid in selecting what tasks, issues, or alternatives to pursue.

Therefore, we can state that there is close relationship between teamwork and decision-making. All decisions, in fact,-in the organizations-should facilitate expansion of business and give more profit, goodwill and prosperity to a business unit.

These decisions may not be efficient when the approach of the decision-maker is casual and superficial. In this, the teamwork can therefore, as we have seen, become a valuable aid for decisional process.

\section{Analysis of the Tenerife Air Disaster and of the Decision Making Dynamics}

This case study is a decision-making process analysis of the aircraft collision which, on March 27, 1977 occurred at Tenerife Island, causing the death of 583 people.

This analysis, will give us the opportunity to verify what has been stated in the research conducted, to confirm that the theoretical results are reflected in the reality of an event.

KLM Flight 4805 and Pan Am Flight 1736, collided on the runway. This collision is an example of how large scale disasters result from errors made by people in crucial decision-making circumstances and illustrates the potentially devastating consequences of ineffective human and organizational behavior. 
This leading case shows the major contributing factors in the accident: stress, small group communication under stress, and small group dynamics and highlights how team resources, i.e. more efficient group dynamics, could probably have avoided these devastating facts.

Tenerife was an unscheduled stop for both flights, their destination in fact was Gran Canaria International Airport, closed due to a bomb planted by the separatist Canary Islands Independence Movement exploded in the terminal, injuring eight people. The civil aviation authorities closed the airport temporarily after the explosion, and all incoming flights bound for Gran Canaria had been diverted to Los Rodeos-Tenerife, in those day a regional airport that could not easily accommodate all of the traffic diverted from Gran Canaria.

The airport had only one runway and one major taxiway running parallel to it, with four short taxiways connecting the two. While waiting for Gran Canaria airport to reopen, the diverted airplanes took up so much space that they were having to park on the long taxiway, making it unavailable for the purpose of taxiing. Instead, departing aircraft needed to taxi along the runway to position themselves for takeoff, a procedure known as a backtaxi or backtrack.

The authorities reopened Gran Canaria airport once the bomb threat had been contained. The Pan Am flight was ready to depart from Tenerife, but was unable to maneuver around the refueling KLM, in order to reach the runway for takeoff, due to a lack of safe clearance between the two planes.

The refueling of KLM took about 35 minutes, after which the passengers were brought back to the aircraft. The search for a missing Dutch family of four, who had not returned to the waiting KLM plane, delayed the flight even further.

Finally, The air traffic control tower instructed the KLM to taxi down the entire length of the runway and then make a 180-degree turn to get into takeoff position.

Because of the tarmac congestion, the normal route to runway is blocked. Departing planes will need to taxi down on the runway itself. Reaching the end, they'll make a 180-degree turn before taking off in the opposite direction.

The airport has no ground tracking radar. While the KLM was backtaxiing on the runway, the controller asked the flight crew to report when it was ready to copy the tower clearance to take off, but, because the flight crew was performing the pre-departure checklist, copying this clearance was postponed until the aircraft was in takeoff position on Runway.

In the meanwhile, is poor the visibility Shortly afterward, the Pan Am was instructed to follow the KLM down the same runway, exit it by taking the third exit on their left and then use the parallel taxiway. There were no markings or signs to identify the runway exits and they were in conditions of poor visibility. The Pan Am crew appeared to remain unsure of their position on the runway until the collision, which occurred near the intersection with the fourth taxiway

KLM first officer, takes the radio and receives the ATC route clearance. This is not a takeoff clearance, but rather a procedure outlining turns, altitudes, and frequencies for use once airborne. Normally it is received well prior to an aircraft 
taking the runway, but the pilots have been too busy with checklists and taxi instructions until now. They are tired, annoyed, and anxious to get going.

The co-pilot, believing that his commander was already proceeding with takeoff, informs him that he has not received takeoff clearance, but the commander appears increasingly nervous and urges him to ask for it.

The pilot warns the control tower that they are about to take off. The ATC probably did not understand whether this meant that the aircraft was stationary and ready to take off or that it had already jumped down the runway, so he was told to wait. Meanwhile, the Pan Am crew, hearing the KLM takeoff announcement, simultaneously called the tower on the same frequency and warned that they are still on the runway.

The resulting overlapping voices generated an interference, as a result of which the KLM did not receive the Pan Am call and at the same time heard the waiting message from the tower in a fragmentary way-perceiving only the takeoff authorization-and ran into the other airplane, causing one of the most serious accidents in aviation history.

The special feature of the disaster that occurred in Tenerife is that there was no technical anomaly, such as an aircraft engine failure, nor was the accident mainly due to bad weather (to be considered as an ancillary event). The responsibility for the accident is entirely attributable to the wrong behavior of the persons involved (absence of teamwork), the decision-making process and the organization to which they belonged.

In the KLM team (commandeer, co-pilot, flight engineer) a series of dynamics and processes that led him to failure are visible. The group was in a highly stressful situation (such as the Pan Am team, the control tower operators and other people involved). This difficult situation led the KLM team to various difficulties.

It is clear that the wrong decisions of the pilot of the aircraft were not contradicted by either the co-pilot or the flight engineer (Weick, 1990) [9]. At the time when the KLM aircraft was preparing for take-off, the co-pilot knew that the pilot was starting the take-off phase incorrectly (i.e., as seen, without authorization).

The commander is completely devoid of situational awareness, and the KLM co-pilot does not try anything to prove his aversion to this behavior. Even the flight engineer had suspicions that the Pan Am aircraft was still on the runway, but he did not insist strongly on convincing the pilot to be more cautious and avoid the risk of an accident.

The KLM team therefore proved to be a group of three members working individually. It seems to have the characteristics of a mechanistic group structure that tends to involve into a bureaucratic hierarchy and formal chains of command and communication. In this type of group the decision-making processes tend to be highly centralized; in the case in question, in fact, the KLM commander, in the stressful situation, isolated himself from the others and took decisions without consulting them, convinced that his decision was the right one 
and that reality confirmed what he had decided (confirmation bias). By analyzing the situation created, we are witnessing a form of groupthink that has enabled the members of the group to minimize conflicts and reach consensus without resorting to a critical evaluation of ideas.

The co-pilot, given his inexperience, avoided contradicting the commander's decisions, probably to avoid the embarrassment or anger of the leader towards him (the same happens for the engineer).

Moreover, in a stressful and critical situation such as the one in which they found themselves, they could not afford to undermine the cohesion of the group; moreover, given the Dutch provisions that obliged them to return to the scheduled monthly flight hours (stress to restart with as little delay as possible), they shared this common objective, which led them to avoid contradicting the decision to take off without authorization.

The whole process of interpretation by the KLM team of the events that were happening and those that would soon happen depends on several factors: language, social interactions between the actors involved, therefore the purely hierarchical relationships that influence attitudes and communication norms in the group [10].

The analysis of the case shows us that there could have been different barriers to prevent the event.

According to studies of Reason (1990), we are faced with an organizational incident, where the lack of team decisions in the process created the conditions for fatal errors.

The Reason's theory, takes its name from the use of a metaphor (Swiss cheese model, Figure 1), where the activities that make up the field under consideration are represented (by slices of gruyere (Swiss cheese) side by side, while the holes represent the events that can individually contribute to the accident (human error, failure of an equipment, lack of maintenance, lack of use or removal of a protection, etc.); as long as the holes do not overlap, the error remains latent, but when the holes in the cheese match the unwanted event occurs.

In other words, the individual mitigation measures (gruyère slices), each with a potential intrinsic weakness, are not sufficient, but the redundancy of safety systems is an essential component in risk mitigation. Several barriers, in this case, did not work, leading to a chain of events [11].

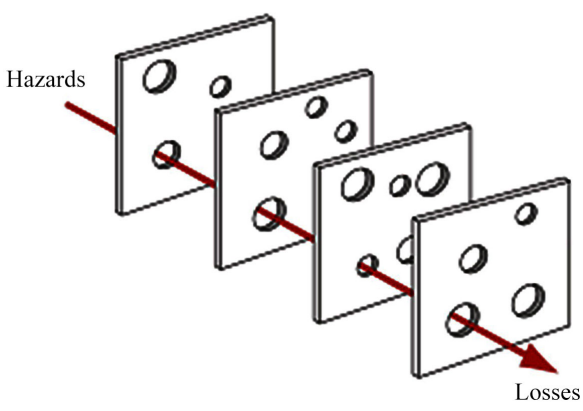

Figure 1. The Swiss cheese model. 
The team that could have helped the decisional process did not work in this case.

If there had been more participation, events would have followed a different path.

\section{Conclusions}

Taking a decision is always a time of great uncertainty. Every time we find ourselves choosing between at least two options, we try to calculate the risk of the action we are taking, estimating the chances of an undesired event.

The individual is not able, in a natural way, to estimate the risk of his choice, since he is influenced by a whole cognitive and cultural background that makes him overestimate or underestimate the value of a possible decision.

Within an organization, wrongly estimating a decision has a much greater impact; the answer is to look in the teamwork for the solution to this possible decisional error.

Complex organizations can therefore benefit from this group approach, where elements that are not taken into account by the individual, can be considered by the group, becoming possible barriers for the prediction of an error.

Different tools can be useful; sharing information, resources or approaches, makes it possible to evaluate options that could not have been identified.

Therefore we can state that, within the decisional process, the teamwork is a fundamental element for the effectiveness of an organization, precisely becausecompared to the single individual-on average the result of the decision-making process of a group exceeds for efficiency that of the most gifted element that is part of it.

In an organization, it is therefore advantageous to work in groups, because the correct management of this element assumes an importance that should not be underestimated.

The efficiency of a group is the result of interaction, discussion and sharing of aims by its members.

From this point of view, on the scale of individual involvement, a certain emotional participation in the common goal is more noticeable in a team than in the occasional group where character aspects can easily emerge that tend to hinder the advantage of working together.

Moreover, given the better organization that groups can give to their decision making activity, it is easier to trigger those factors that benefit the quality of the decision: the subdivision of activities is possible when several people dedicate themselves to the same task, dividing a complex work into parts that are easier to assign to individual members; the filter effect that occurs when the group deliberately ignores certain information available to it, in order to focus attention on the most important aspects of the decision, favoring the best choice; the compensation, for which it is more convenient to rely on the combination of the estimates of many people, rather than a single one, in order to mitigate the ex- 
tremes and to arrive at a more likely average value; the adherence that occurs among the members of the group, partly due to the sharing of the knowledge of its participants-although this is a positive factor only if it does not prevent the search for alternatives.

\section{Conflicts of Interest}

The author declares no conflicts of interest regarding the publication of this paper.

\section{References}

[1] Tabassi, A.A., Ramli, M. and Bakar, A.H.A. (2012) Effects of Training and Motivation Practices on Team Work Improvement and Task Efficiency. The Case of Construction Firms. International Journal of Project Management, 30, 213-224. https://doi.org/10.1016/j.ijproman.2011.05.009

[2] Negulescua, O. and Dovalb, E. (2014) The Quality of Decision Making Process Related to Organizations' Effectiveness. Procedia Economics and Finance, 15, 858-863. https://doi.org/10.1016/S2212-5671(14)00548-6

[3] Baddeley, A.D. and Hitch, G.J. (1974) Working Memory. In: Bower, G.H., Ed., The Psychology of Learning and Motivation, Academic Press, New York, 47-89. https://doi.org/10.1016/S0079-7421(08)60452-1

[4] De Andreis, F., Fredella, N., Castelli, B. and De Marco, S. (2019) The Instruments of Risk Management as an Opportunity for the Healthcare Organizations. International Journal of Management Sciences and Business Research, 8, 35-40.

[5] Giddens, A. (1999) Risk and Responsibility. Modern Law Review, 62, 1-10. https://doi.org/10.1111/1468-2230.00188

[6] De Andreis, F. and Florio, M. (2019) Risk Management Instruments, Strategies and Impacts in the Complex Organizations. American Journal of Industrial and Business Management, 9, 1157-1167. https://doi.org/10.4236/ajibm.2019.95078

[7] Nickerson, R.S. (1998) Confirmation Bias: A Ubiquitous Phenomenon in Many Guises. Review of General Psychology, 2, 1175-220.

https://doi.org/10.1037/1089-2680.2.2.175

[8] Guzzo, R.A. and Salas, E. (1995) Team Effectiveness and Decision Making in Organizations. Pfeiffer \& Co.

[9] Weick, K.E. (1990) The Vulnerable System: An Analysis of the Tenerife Air Disaster. Journal of Management, 16, 571-593. https://doi.org/10.1177/014920639001600304

[10] Orasanu, J. and Connolly, T. (1993) The Reinvention of Decision Making. In: Klein, G.A., Orasanu, J., Calderwood, R. and Zsambok, C.E., Eds., Decision Making in Action: Models and Methods, Ablex, Norwood, 3-20.

[11] Reason, J. (1990) Managing the Risks of Organizational Accidents. Ashgate, Farnham. 